\title{
Regularity of Weak Solutions to Elliptic Problem with Irregular Data
}

\author{
Abdelaziz Hellal ${ }^{1}$ \\ ${ }^{1}$ Mohamed Boudiaf University of M'Sila. Faculty of Mathematics and Informatics
}

January 4, 2022

\begin{abstract}
This paper is concerned with the study of the nonlinear elliptic equations in a bounded subset $\Omega$ [?] RN Au =f, where A is an operator of Leray-Lions type acted from the space W1, $\mathrm{p}(\cdot) 0(\Omega)$ into its dual. when the second term $\mathrm{f}$ belongs to Lm $(\cdot)$, with $\mathrm{m}(\cdot)>1$ being small. we prove existence and regularity of weak solutions for this class of problems $\mathrm{p}(\mathrm{x})$-growth conditions. The functional framework involves Sobolev spaces with variable exponents as well as Lebesgue spaces with variable exponents.
\end{abstract}

\section{Hosted file}

Article_Abdelaziz Hellal.pdf available at https://authorea.com/users/453836/articles/551621regularity-of-weak-solutions-to-elliptic-problem-with-irregular-data 